\title{
Laparoscopic Roux-en-Y gastric bypass with ileal transposition - an alternative surgical treatment for type 2 diabetes mellitus and gastroesophageal reflux
}

\author{
Feyzullah Ersoz ${ }^{1}$, Yigit Duzkoylu' ${ }^{1}$, Mehmet Mehdi Deniz ${ }^{1}$, Mustafa Boz ${ }^{2}$ \\ ${ }^{1}$ General Surgery Clinic, Istanbul Education and Research Hospital, Istanbul, Turkey \\ ${ }^{2}$ Diabetes and Endocrinology Department, Istanbul Education and Research Hospital, Istanbul, Turkey
}

Videosurgery Miniinv 2015; 10 (3): 481-485

DOI: $10.5114 /$ wiitm.2015.54224

\begin{abstract}
Currently, type 2 diabetes mellitus (T2DM) is considered as a gastrointestinal disease. Numerous studies have demonstrated that bariatric operations ameliorate T2DM significantly. The laparoscopic Roux-en- $Y$ gastric bypass (L-RYGB) is considered as the gold standard procedure. We aimed to evaluate the feasibility and impact of L-RYGB with ileal transposition (or interposition) on diabetes resolution in a patient who has not benefited from any medical therapy, with its additional effect on the amelioration of gastroesophageal reflux disease. The patient was a 38-yearold diabetic woman with uncontrolled blood glucose despite medical treatment, with additional gastroesophageal reflux disease. Following the procedure, her biochemical parameters and reflux symptoms improved significantly within 10 months. We think that L-RYGB with ileal transposition may be easily employed to gain a maximum effect in diabetics with adverse prognosis. This technique may be an alternative in the treatment of type 2 diabetic patients with gastroesophageal reflux.
\end{abstract}

Key words: gastroesophageal reflux disease, diabetes mellitus, obesity surgery, metabolic surgery, Roux-en-y gastric bypass, ileal transposition-interposition.

\section{Introduction}

Obesity and diabetes are the twin epidemics of the twenty first century. Obesity, particularly central obesity, is closely associated with insulin resistance and type 2 diabetes mellitus (T2DM). Despite the recent advances in pharmacology and the introduction of multi-agent treatment modalities, a large number of patients still do not have optimal results [1].

The efficacy of bariatric surgery in adjusting and even normalizing glucose levels in obese patients with T2DM has been confirmed in numerous observational studies. Remission of diabetes can be achieved in more than $60 \%$ of patients after bariatric surgery, with significant variability between different surgical procedures [2].

Recent studies have shown the positive effects of modified bariatric surgery procedures in the treatment of non-obese T2DM patients. Therefore, bariatric surgery is also referred to as metabolic surgery because of its results ranging from weight loss to metabolic control, especially in patients with T2DM $[3,4]$. Currently, T2DM can be considered as a gastrointestinal disease that can be treated surgically, but there is no consensus yet on the criteria of patient selection as various techniques using different mechanisms have different remission rates.

\section{Address for correspondence}

Assoc. Prof. Feyzullah Ersoz, General Surgery Clinic, Istanbul Education and Research Hospital, Samatya, Fatih 34000 Istanbul, Turkey, phone: +90 2124590000, e-mail: feyzullahersoz@gmail.com 
Malabsorptive operations, particularly with biliopancreatic diversion, have the capability of inducing the release of anorexigenic neuropeptides since the whole jejunum is bypassed and food passes directly to the ileum [5]. With the understanding that these neuropeptides have positive effects on weight loss with ileal interpositioning, the standard procedures have been modified to achieve maximum remission with minimal long-term complications. Thus, bariatric surgery has evolved into metabolic/ diabetes surgery, and it is still evolving, for there will always be a search for better surgical techniques.

The laparoscopic Roux-en-Y gastric bypass (L-RYGB) is considered the gold standard among bariatric procedures. Although this method leads to substantial remission of T2DM in up to $60-84 \%$ of cases and regarding glycemic control and clinical findings, previous studies concerning RYGB have shown that old age, longer duration of diabetes, lower levels of C-peptide and lower body mass index (BMI) reduce the chances of a positive response to surgery [6].

We aimed to evaluate the feasibility and impact of L-RYGB with ileal transposition on the management of diabetes in a patient who has not benefited from any medical therapy. The procedure also had the added benefit of alleviating the symptoms of gastroesophageal reflux disease. To the best of our knowledge, this is the first report on this subject in the literature.

\section{Case report}

A 38-year-old female patient had been referred to general surgery from the endocrinology department because of uncontrolled blood glucose. She had had diabetes for 16 years and had been using insulin for 12 years. She was also under treatment for hypertension, dislipidemia and gastroesophageal reflux disease. Her medications included daily metformin $(2 \times 1000 \mathrm{mg})$, insulin aspart $(3 \times 20 \mathrm{IU})$ and insulin glargine $(1 \times 40 \mathrm{IU})$, amlodipine $(1 \times 10 \mathrm{mg})$, metoprolol $(1 \times 50 \mathrm{mg})$, acetylsalicylic acid $(1 \times 100 \mathrm{mg})$, atorvastatin $(1 \times 10 \mathrm{mg})$ and pantoprazole $(1 \times 40 \mathrm{mg})$ at the time of admission. Her BMI was $44 \mathrm{~kg} / \mathrm{m}^{2}$. Liver and kidney function tests were found to be normal. Her blood pressure was 145/90 mm Hg, $\mathrm{HbA}_{1 \mathrm{c}}$ $10.5 \%$, total cholesterol $241 \mathrm{mg} / \mathrm{dl}$, high-density lipoprotein (HDL) cholesterol $40 \mathrm{mg} / \mathrm{dl}$, low-density lipoprotein (LDL) cholesterol $160 \mathrm{mg} / \mathrm{dl}$, triglyceride $225 \mathrm{mg} / \mathrm{dl}$, hemoglobin $12.3 \mathrm{~g} / \mathrm{dl}$ and C-peptide
$1.4 \mathrm{ng} / \mathrm{dl}$. Upper endoscopy revealed grade B reflux esophagitis.

Thus we decided to perform an L-RYGB with ileal transposition (or interposition) so as to provide a better remission of diabetes compared to alternative procedures of bariatric surgery.

In our clinic, L-RYGB operations are performed using the fully stapled technique introduced by Dillemans et al. [7].

The gastric pouch was created (Figure $1 \mathrm{~A}$ ) with blue cartridges (Ethicon, USA), the lower left corner of the pouch was cut, and a purse-string suture was placed using a monofilament_suture. A $25 \mathrm{~mm}-$ $3.5 \mathrm{~mm}$ circular stapler with DST series (Covidien, USA) was then introduced intra-abdominally through the most left lateral, manually dilated trocar site. The anvil was brought into the gastric pouch opening and the purse string was tied. The greater omentum was divided. Later, one more $5-\mathrm{mm}$ port was located at the left lower quadrant using the left lateral Trendelenburg position.

The ileocecal valve was identified and an ileal loop was antecolically pulled up from this point to the gastric pouch. An enterotomy was created at $180 \mathrm{~cm}$ distance from the ileocecal valve and through this opening the circular stapler was introduced over a $5 \mathrm{~cm}$ segment in the jejunal loop distally. Later, we perforated the jejunum and connected the stapler to the anvil, making the anastomosis. The remaining small bowel loop with the previously created opening was then transected at $1 \mathrm{~cm}$ distance proximal to the gastro-jejunostomy using a linear stapler with a $60-\mathrm{mm}$ white cartridge (Ethicon, USA). Thus, the proximal end of the ileal segment was anastomosed to the gastric pouch (Figure $1 \mathrm{~B}$ ).

Then, the end of the long biliopancreatic limb was anastomosed to the terminal ileum $30 \mathrm{~cm}$ proximal from the ileocecal valve, creating a side-to-side enteroenterostomy using a 60- $\mathrm{mm}$ white cartridge, and the distal ileum was transected $30 \mathrm{~cm}$ proximal to the ileocecal valve also using a $60-\mathrm{mm}$ white cartridge (Figure $1 \mathrm{~B}$ ). The distal end of the ileal segment was anastomosed to a point in the jejunum at $50-\mathrm{cm}$ distance from the ligament of Treitz, creating a sideto-side entero-enterostomy, using a linear stapler with a $60-\mathrm{mm}$ white cartridge (Figure $1 \mathrm{~B}$ ). Thereby, at $30 \mathrm{~cm}$ distance from the ileocecal valve, an ileal segment of $150 \mathrm{~cm}$ was relocated in the isoperistaltic direction. The proximal end of this segment was anastomosed to the gastric pouch while the distal 
A

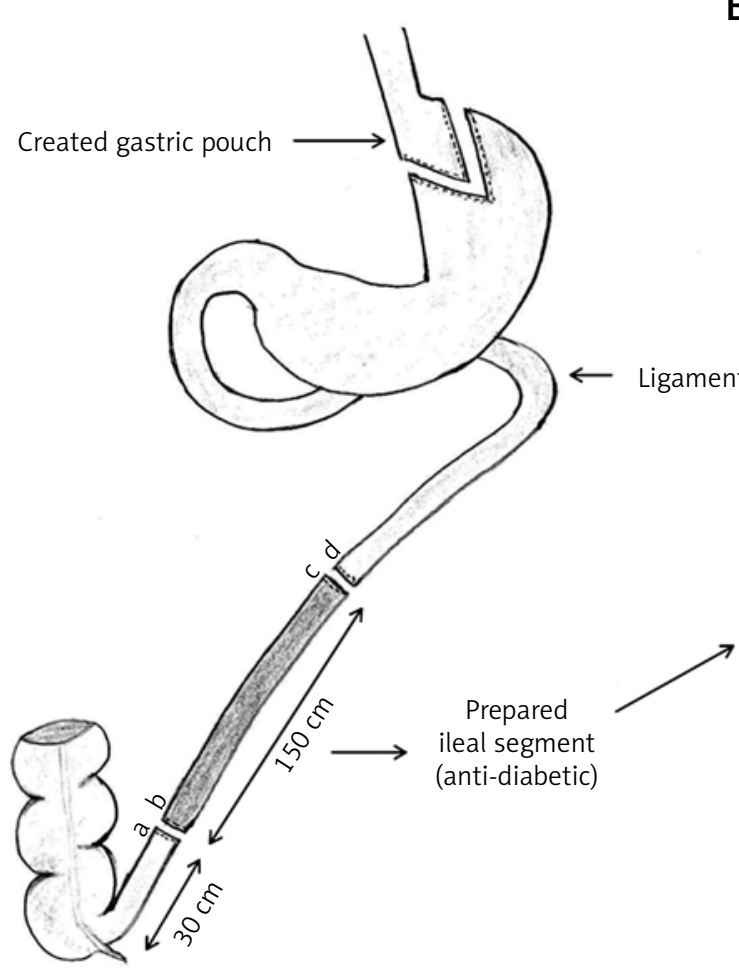

B

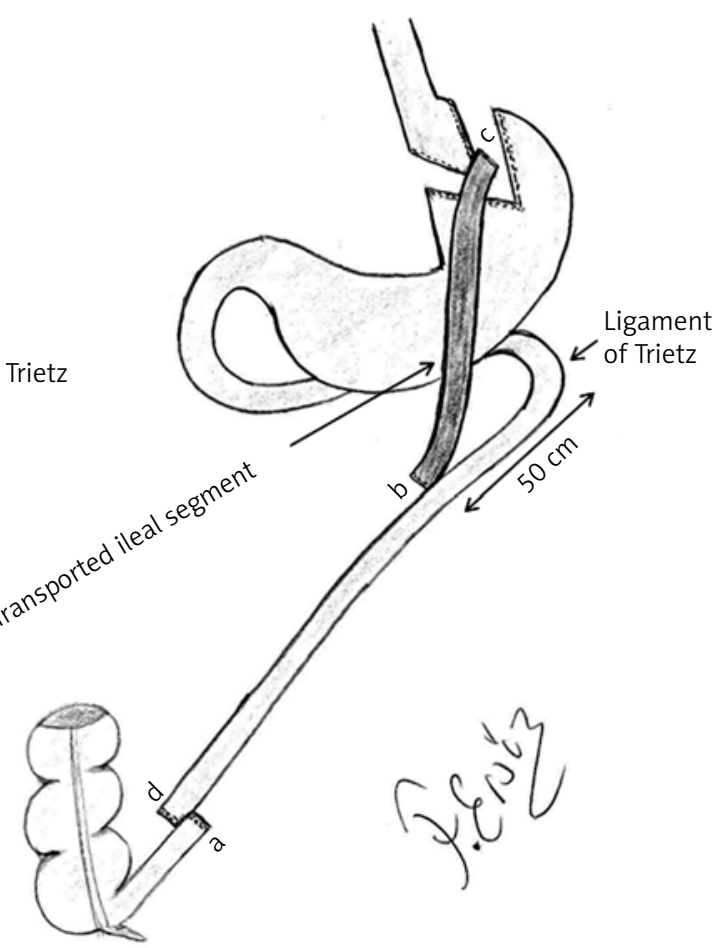

Figure 1. A - Preparation of gastric pouch and ileal segment. B - Roux-n-Y bypass with ileal transposition

end was anastomosed at $50 \mathrm{~cm}$ distance from the ligament of Treitz (Figure 1 B). Following hemostasis, a drainage tube was placed near the gastric pouch and the operation was completed.

Unlike Dillemens et al., who performed the procedure with an average operational time of 60 90 min, we applied L-RYGB with ileal transposition using the same technique with an extra anastomosis in nearly $220 \mathrm{~min}$, which may be due to the fact that it was our first application of the procedure.

With a low dose insulin therapy, the patient was discharged 4 days after the surgery without any complications. At 10 months after the surgery, she had lost $31 \mathrm{~kg}$ (23.6\% loss) and BMI had decreased to $26 \mathrm{~kg} / \mathrm{m}^{2}$. The $\mathrm{HbA}_{1 \mathrm{c}}$ was $4.8 \%$, total cholesterol was $162.5 \mathrm{mg} / \mathrm{dl}$, HDL $58 \mathrm{mg} / \mathrm{dl}$, LDL cholesterol $85 \mathrm{mg} / \mathrm{dl}$, triglyceride $98 \mathrm{mg} / \mathrm{dl}$. Symptoms of gastroesophageal reflux had disappeared without any medical therapy.

\section{Discussion}

Type 2 diabetes mellitus is considered as a chronic and progressive disease because of the fact that pharmacological treatment cannot ensure a cure, but only delay the development of its complications. A significant amount of evidence has demonstrated that bariatric operations, particularly procedures bypassing the duodenum and part of the jejunum, rapidly and significantly ameliorate T2DM, leading to remission of the disease in the majority of cases.

A dramatic improvement in glucose control frequently occurs immediately after bariatric surgery, usually within 1 week, before significant weight loss. Therefore, the mechanism of immediate remission of diabetes or improvement in disease control appears to be weight loss-independent [8]. To understand this mechanism better, it is crucial to investigate the importance of intestinal hormones and their effects.

The difference in insulin secretion following intravenous versus oral glucose administration is referred to as the incretin effect [9]. This effect is mostly mediated by glucagon-like peptide-1 (GLP-1) and glucose-dependent insulinotropic peptide (GIP). Both stimulate insulin secretion in a glucose-dependent manner and are thus called as incretins. In T2DM, the incretin effect is markedly decreased [10]. 
Glucagon-like peptide- 1 is derived from the transcription product of the proglucagon gene. Being an intestinal hormone, it is chiefly secreted by intestinal $L$ cells. It increases the glucose sensitivity of pancreatic B-cells, inhibits B cell apoptosis, and stimulates the proliferation and differentiation of $B$ cells. Glucagon-like peptide- 1 also inhibits gastric acid secretion and motility, which delays carbohydrate absorption and contributes to the satiating effect.

Glucagon-like peptide is synthesized by $\mathrm{K}$ cells, which are found in the mucosa of the duodenum and the upper jejunum, and it is also known as the gastric inhibitory polypeptide. It has been shown that diabetics are not responsive to GIP, demonstrating lower levels of GIP secretion after a meal compared to non-diabetics.

Among the procedures of bariatric surgery, two procedures, namely the RYGB and the biliopancreatic diversion (BPD), are more effective treatment options for diabetic patients compared to other procedures [5]. Roux-en- $Y$ gastric bypass combines gastric restriction with bypassing of the stomach and the proximal intestine. It is primarily a restrictive procedure and does not typically involve substantial malabsorption unless significant lengths of intestine are bypassed, as seen with the long duodenojejunal limb gastric bypass [11]. One of the components of RYGB is the exclusion of the duodenum and the proximal jejunum from the passage of food, known as the "foregut hypothesis".

In nonobese type 2 diabetic animal models, such as Goto-Kakizaki rats, bypassing the duodenum with the proximal jejunum probably increases GIP secretion by decreasing the activity of GIP inhibitor hormones [12]. It has been found that diabetics who are not responsive to GIP have lower levels of GIP secretion after a meal when compared to non-diabetics.

Another component of the RYGB is the "hindgut hypothesis". The rapid delivery of nutrients has been demonstrated to stimulate the $L$ cells in the distal intestine to secrete incretin, thus enhancing insulin secretion and sensitivity. The potential mediators of this effect are GLP-1, GIP, peptide YY (PYY) and oxyntomodulin. According to this hypothesis, the faster the nutrients reach the distal ileum, the greater is the hindgut effect, because $L$ cells are found mainly in the distal ileum and create the incretin effect following contact with nutrients [13].

The BPD procedure is profoundly effective in the remission of diabetes. Although it is similar to RYGB in principle, the gastric pouch is larger $(250-500 \mathrm{ml})$, the alimentary limb is longer (approximately $250 \mathrm{~cm}$ ) and the common limb is shorter (approximately $50 \mathrm{~cm}$ ). Kaska et al. found that the length of the common limb has a significant effect on the remission of T2DM, which is more than the length of the biliary limb [14]. The foregut effect of BPD is similar to RYGB, but the incretin effect is more evident because nutrients reach the distal ileum faster, which increases the hindgut effect. This may lead to amelioration in diabetic patients with a rate of $100 \%$. Unfortunately, this technique is highly malabsorptive, leading to protein, vitamin and mineral deficiency as well as persistent diarrhea. Therefore, it is rarely performed today.

To decrease the malabsorptive effect of BPD, Marceau et al. introduced the BPD with duodenal switch (BPD-DS) procedure in 1998 [15]. This technique is both restrictive and malabsorptive. Although it is effective in diabetes remission, the laparoscopic technique is very complex and may still cause diarrhea and malnutrition, just not as much as BPD does.

DePaula et al. performed sleeve gastrectomy with ileal transposition interposition (II-SG) for the first time in humans [16] to gain maximum advantage from the hindgut effect without causing malabsorption. Later, he performed ileal interposition combined with a diverted sleeve gastrectomy (II-DSG) to also benefit from the foregut effect and reported that diverted ileal interposition is more effective in diabetes remission because of the additional foregut effect. In II-DSG, nutrients reach the distal ileum very rapidly, creating a strong hindgut effect, and it has been reported to be very effective even in patients with low $\mathrm{BMI}$. It has also been reported not to cause malabsorption due to the much longer common limb.

Despite its effectiveness, this technique is not performed frequently because of the complex design and difficulties in laparoscopic practice. Additionally, pulling the sleeved gastric pouch down to the lower abdomen impairs the integrity of the intraabdominal anatomy. In the presence of disorders in common hepatic ducts, it is almost impossible to perform endoscopic retrograde cholangiopancreatography (ERCP) following II-DSG. Conversely, in RYGB, it is possible to perform hand-assisted ERCP through the remnant gastric pouch [17], which is an advantage of L-RYGB with ileal transposition over II-DSG.

We have found that II-DSG causes a lesser hindgut effect due to the pyloric valve when compared to 
L-RYGB with ileal transposition, because of the fact that the nutrients reach the transposed ileum segment later and in lower amounts with the former technique, since sleeve gastrectomy is an obstructive method. In II-DSG, the duodenal stump after the pylorus is anastomosed to the transposed ileum segment; we think that nutrients can enter a very short segment of duodenum, which may decrease the foregut effect of the procedure. Conversely, in L-RYGB with ileal transposition, the foregut effect is maximized, as the duodenum is completely bypassed. Additionally, the dumping syndrome that may develop after the ingestion of foods with high glucose levels may be a protective factor in L-RYGB with ileal transposition.

The incidence of gastroesophageal reflux disease may increase following II-DSG, which might have worsened esophagitis in our patient, who already had grade B esophagitis. Thus, we preferred RYGB because of its anti-reflux effect.

\section{Conclusions}

We think that L-RYGB with ileal transposition may be performed by surgeons experienced in the procedure to gain benefit in diabetics with adverse prognostic features. In the future, this technique may be an alternative in the treatment of type 2 diabetic patients with gastroesophageal reflux, but more comprehensive prospective studies including large patient groups are needed to evaluate the results of our technique and its clinical efficacy.

\section{Conflict of interest}

The authors declare no conflict of interests.

\section{References}

1. Lebovitz H. Type 2 diabetes mellitus, current therapies and emergence of surgical options. Nat Rev 2011; 10: 1038.

2. Ferrannini E, Mingrone G. Impact of different bariatric surgical procedures on insulin action and beta-cell function in type 2 diabetes. Diabetes Care 2009; 32: 514-20.

3. Jastrzębska-Mierzynska M, Ostrowska L, Hady HR, et al. The impact of bariatric surgery on nutritional status of patients. Videosurgery Miniinv 2015; 10: 115-24.

4. Greenway SE, Greenway FL 3rd, Klein S. Effects of obesity surgery on non-insulin dependent diabetes mellitus. Arch Surg 2002; 137: 1109-17.

5. Buchwald H, Avidor Y, Braunwald E, et al. Bariatric surgery: systematic review and meta-analysis. J Am Med Assoc 2004; 292 1724-37.

6. Still CD, Wood GC, Benotti P, et al. Preoperative prediction of type 2 diabetes remission after Roux-en-Y gastric bypass sur- gery: a retrospective cohort study. Lancet Diabetes Endocrinol 2014; 2: 38-45.

7. Dillemans B, Sakran N, Van Cauwenberge S, et al. Standardization of the fully stapled laparoscopic Roux-en-Y gastric bypass for obesity reduces early immediate postoperative morbidity and mortality: a single center study on 2606 patients. Obes Surg 2009; 19: 1355-64

8. Pories WJ, Swanson MS, MacDonald KG, et al. Who would have thought it? An operation proves to be the most effective therapy for adult-onset diabetes mellitus. Ann Surg 1995; 222: 339-50.

9. Creutzfeldt W. The incretin concept today. Diabetologia 1979; 16: 75-85.

10. Speck M, Cho YM, Asadi A, et al. Duodenal-jejunal bypass protects GK rats from \{beta\}-cell loss and aggravation of hyperglycemia and increases enteroendocrine cells coexpressing GIP and GLP-1. Am J Physiol Endocrinol Metab 2011; 300: E923-32.

11. Brolin RE, LaMarca LB, Kenler HA, Cody RP. Malabsorptive gastric bypass in patients with superobesity. I Gastrointest Surg 2002; 6: 195-204

12. Rubino F, Marescaux J. Effect of duodenal-jejunal exclusion in a non-obese animal model of type 2 diabetes: a new perspective for an old disease. Ann Surg 2004; 239: 1-11.

13. Orskov C, Wettergren A, Holst JJ. Secretion of the incretin hormones glucagon-like peptide- 1 and gastric inhibitory polypeptide correlates with insulin secretion in normal man throughout the day. Scand I Gastroenterol 1996; 31: 665-70.

14. Kaska L, Kobiela J, Proczko M, et al. Does the length of the biliary limb influence medium-term laboratory remission of type 2 diabetes mellitus after RouX-en-Y gastric bypass in morbidly obese patients? Videosurg Miniinv 2014; 9: 31-9.

15. Marceau P, Biron S, Hould FS, et al. Duodenal switch improved standard biliopancreatic diversion: a retrospective study. Surg Obes Rel Dis 2009; 5: 43-7.

16. DePaula AL, Macedo AL, Mota BR, et al. Laparoscopic ileal interposition associated to a diverted sleeve gastrectomy is an effective operation for the treatment of type 2 diabetes mellitus patients with BMI 21-29. Surg Endosc 2009; 23: 1313-20.

17. Facchiano E, Quartararo G, Pavoni V, et al. Laparoscopy-assisted transgastric endoscopic retrograde cholangiopancreatography (ERCP) after Roux-en-Y gastric bypass: technical features. Obes Surg 2015; 25: 373-6.

Received: 20.04.2015, accepted: 19.08.2015. 